\title{
AIP
}

\section{Probing inhomogeneous composition in core/shell nanowires by Raman spectroscopy}

F. Amaduzzi, E. Alarcón-Lladó, E. Russo-Averchi, F. Matteini, M. Heiß, G. Tütüncüoglu, S. Conesa-Boj, M. de la

Mata, J. Arbiol, and A. Fontcuberta i Morral

Citation: Journal of Applied Physics 116, 184303 (2014); doi: 10.1063/1.4901504

View online: http://dx.doi.org/10.1063/1.4901504

View Table of Contents: http://scitation.aip.org/content/aip/journal/jap/116/18?ver=pdfcov

Published by the AIP Publishing

\section{Articles you may be interested in}

Raman spectroscopy of GaP/GaNP core/shell nanowires

Appl. Phys. Lett. 105, 193102 (2014); 10.1063/1.4901446

Polarization dependent photocurrent spectroscopy of single wurtzite GaAs/AIGaAs core-shell nanowires Appl. Phys. Lett. 102, 142107 (2013); 10.1063/1.4801865

Ultraviolet Raman spectroscopy of GaN/AIN core-shell nanowires: Core, shell, and interface modes Appl. Phys. Lett. 102, 143109 (2013); 10.1063/1.4801779

Polarization anisotropy of individual core/shell GaAs/AIGaAs nanowires by photocurrent spectroscopy Appl. Phys. Lett. 98, 153106 (2011); 10.1063/1.3578189

Structural and optical analysis of GaAsP/GaP core-shell nanowires

J. Appl. Phys. 106, 124306 (2009); 10.1063/1.3269724

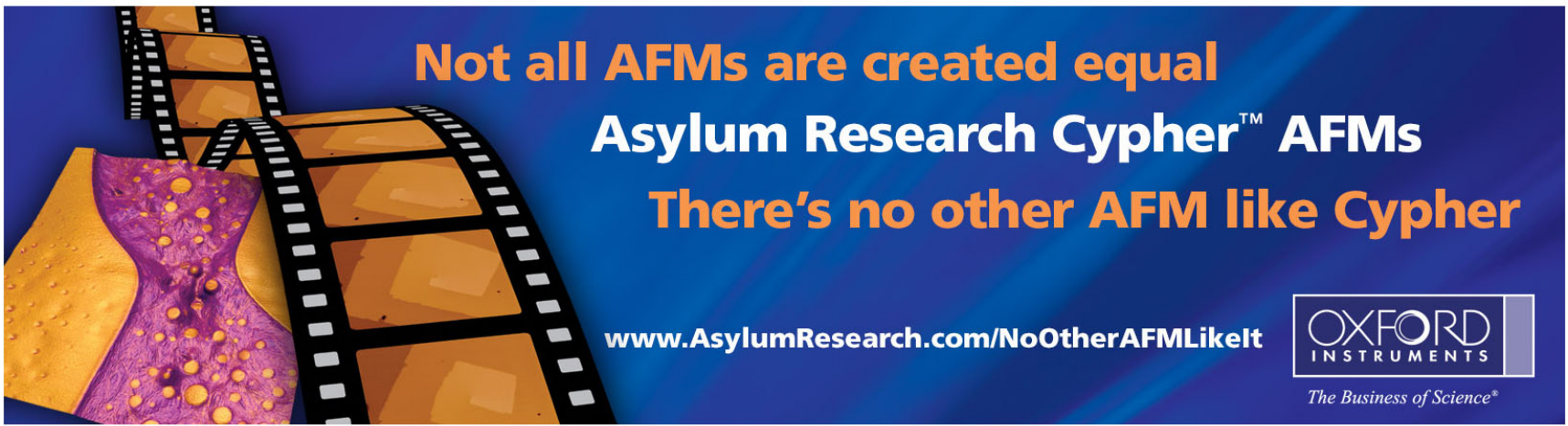




\title{
Probing inhomogeneous composition in core/shell nanowires by Raman spectroscopy
}

\author{
F. Amaduzzi, ${ }^{1}$ E. Alarcón-Lladó, ${ }^{1}$ E. Russo-Averchi, ${ }^{1}$ F. Matteini, ${ }^{1}$ M. Heiß, ${ }^{1}$ \\ G. Tütüncüoglu, ${ }^{1}$ S. Conesa-Boj, ${ }^{1}$ M. de la Mata, ${ }^{2}$ J. Arbiol, ${ }^{2,3}$ and A. Fontcuberta i Morral ${ }^{1}$ \\ ${ }^{1}$ Laboratoire des Matériaux Semiconducteurs, École Polytechnique Fédérale de Lausanne, 1015 Lausanne, \\ Switzerland \\ ${ }^{2}$ Institut de Ciència de Materials de Barcelona (ICMAB-CSIC), Campus de la UAB, 08193 Bellaterra, CAT, \\ Spain \\ ${ }^{3}$ Institució Catalana de Recerca i Estudis Avançats (ICREA), 08019 Barcelona, CAT, Spain
}

(Received 18 September 2014; accepted 31 October 2014; published online 11 November 2014)

\begin{abstract}
Due to its non-destructive and its micro-spatial resolution, Raman spectroscopy is a powerful tool for a rapid structural and compositional characterization of nanoscale materials. Here, by combining the compositional dependence of the Raman peaks with the existence of photonic modes in the nanowires, we address the composition inhomogeneities of $\mathrm{Al}_{x} \mathrm{Ga}_{1-x} \mathrm{As} / \mathrm{GaAs}$ core/shell structures. The experimental results are validated with complementary chemical composition maps of the nanowire cross-sections and finite-difference time-domain simulations of the photonic modes. (C) 2014 AIP Publishing LLC. [http://dx.doi.org/10.1063/1.4901504]
\end{abstract}

\section{INTRODUCTION}

Semiconductor nanowires are filamentary crystals with a tailored diameter between few and few hundred nanometers. Thanks to their geometry, they have inspired new avenues in a large variety of applications. ${ }^{1-10}$ In principle, nearly any material can be synthetized in a bottom-up manner in the nanowire form following the vapor-liquid-solid mechanism or similar. ${ }^{11-16}$ Nanowire-based structures can be further sophisticated by producing heterostructures in the axial, radial, and branched directions. ${ }^{17-24}$ One of the most interesting challenges in the synthesis of nanowires is the fabrication of ternary and quaternary alloys. Being the surface-to-volume ratio enhanced, capillary forces, adatom diffusivities, and surface energies amplify alloy segregation processes. ${ }^{25,26}$ The composition control at the atomic and nanoscale of ternary and quaternary materials in the form of nanowire has not yet reached maturity. In order for this area to progress, techniques that enable a fast and accurate assessment of the composition and its homogeneity are needed. Extremely precise but yet costly techniques, such as atom probe tomography, provide three dimensional information on the nanowire composition down to $0.01 \% .{ }^{27}$ Less costly techniques include high resolution EELS (Electron Energy Loss Spectroscopy) and EDX (Energy Dispersive X-Ray Spectroscopy) in a transmission electron microscope. One inconvenience of these techniques is that they destroy the specimen and probe only a small fraction of it. An alternative non-destructive technique which allows multiple nanowire sampling in a fast manner is the measurement of the phonon spectra by Raman spectroscopy. ${ }^{28-30}$

The optical phonons in a ternary $A l_{x} G a_{1-x} A s$ alloy exhibit a two-mode behavior. Two sets of longitudinal optical (LO) and transversal optical (TO) phonons are observed. Their frequencies vary continuously from those of GaAs(AlAs) to the impurity mode in the other end-member binary $\mathrm{AlAs}(\mathrm{GaAs})$, respectively. The random-element- isodisplacement model proposed by Chen et al. ${ }^{31}$ explains this two mode behavior. Parayanthal et al. ${ }^{28}$ used it to characterize both the epitaxial layer and the interface of $\mathrm{Al}_{x} \mathrm{Ga}_{1-x} \mathrm{As} / \mathrm{GaAs}$ heterojunctions to obtain information on Al composition from the frequency position of the phonon peaks. In this way, Raman frequencies of phonon modes can be used to monitor the composition in a complicated multilayer structure, and the relative Raman intensities can be related to variations in composition. ${ }^{32}$

Raman spectroscopy has also recently been used for the characterization of crystal phase and chemical composition of III-V nanowires. ${ }^{30,33-35}$ Due to the one-dimensional geometry of the nanowire and the dielectric mismatch with the surrounding medium, the bulk Raman selection rules are slightly modified leading to a strong dependence on the polarization angle between the incident electric field and the nanowire axis. ${ }^{36}$ In particular, the intensity of the scattered mode is higher for incident and scattered light with polarization parallel to the nanowire axis. This effect is often called antenna effect. ${ }^{37}$ It is also observed in absorption and luminescence studies. ${ }^{38-40}$ Recently, resonant photonic effects leading to increased absorption have been demonstrated in nanowires. ${ }^{2,41,42}$ These optical resonances in nanowires have shown to play an important role in the non-linear optical properties of nanowires, such as Raman scattering and second harmonic generation. ${ }^{43-47}$ As an example, Cao et $a l .{ }^{43}$ have demonstrated a strong enhancement of the spontaneous Raman scattering from individual silicon nanowires, which depends in a very strong manner on the diameter, excitation wavelength, and incident polarization.

In this work, we investigate the use of Raman spectroscopy to determine the chemical composition of GaAs/ $\mathrm{Al}_{x} \mathrm{Ga}_{1-x} \mathrm{As}$ core/shell nanowires. As a consequence of the wavelength dependence of photonic modes, incident light probes different regions of the structure in a non-intuitive manner and Raman spectra strongly depend on the excitation 
wavelength. This demonstrates the importance of theoretical simulations on the distribution of the electromagnetic field inside the nanowires for the understanding of Raman spectroscopy experiments on nanowires.

\section{EXPERIMENT}

The GaAs $/ \mathrm{Al}_{x} \mathrm{Ga}_{1-x} \mathrm{As}$ core/shell nanowire structures were grown by molecular beam epitaxy in a DCA P600 MBE machine on a 2-in. Si(111) substrate. The core was obtained by the gallium-assisted method as reported in Refs. 48 and 49. After stopping the axial growth, the conditions were switched from axial to radial growth in order to obtain an $\mathrm{Al}_{x} \mathrm{Ga}_{1-x} \mathrm{As}$ shell with different thicknesses and nominal $\mathrm{Al}$ compositions of $x=25 \%, 50 \%$, and $70 \%$, as described in Refs. 26 and 50. The $\mathrm{Al}_{x} \mathrm{Ga}_{1-x}$ As shell was capped with $5 \mathrm{~nm}$ of GaAs to prevent oxidation upon exposure to air. The exact growth protocol is reported in the Supporting Information. $^{51}$

Compositional mappings of the nanowires were performed on cross-sections prepared by microtomy. ${ }^{25,52}$ The structure and composition were characterized by combining high-angle annular dark field scanning transmission electron microscopy (STEM) and EDS or EDX in Figure 1. From the different contrasts, we can distinguish between the GaAs regions (lighter contrast) and $\mathrm{Al}_{x} \mathrm{Ga}_{1-x} \mathrm{As}$ regions (darker contrast). In the rightmost image, the contrast is not uniform because the cross-section lamella was thinner than for the other samples. The embedding resin in the thinner lamellas is more susceptible to beam-induced heating. Heating of the resin can result in slight tilting of the sample and thus a distorted contrast due to additional backscattering. The nanowires exhibit a hexagonal cross-section with side facets pertaining to the $\{110\}$ family. Al inhomogeneities are deduced from the presence of dark stripes at the corners. This has been previously observed and reported in other works. ${ }^{25,26,53}$

The Raman scattering measurements were performed on single nanowires at room temperature. The 488.0, 520.8, and $647.1 \mathrm{~nm}$ lines of an $\mathrm{Ar}-\mathrm{Kr}^{+}$laser were used as excitation. The laser light incident with a power of $\approx 300 \mu \mathrm{W}$ was focused on the nanowire with a microscope objective with numerical aperture $N A=0.75$. The scattered light was analyzed by a TriVista triple spectrometer and detected by a liquid nitrogen cooled multichannel charge-coupled-device (CCD)

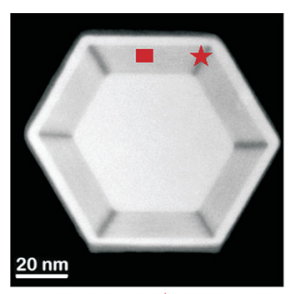

$25 \% \mathrm{Al} \star 47 \% \mathrm{Al}$

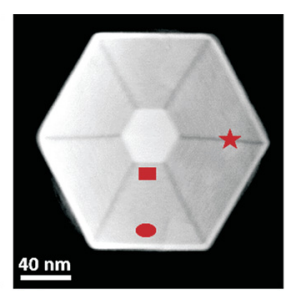

$40-47 \% \mathrm{Al} \star 60 \% \mathrm{Al}$

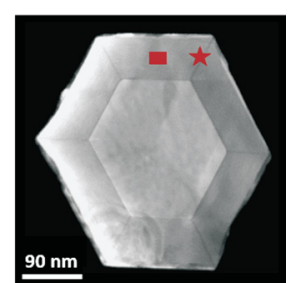

- $70 \% \mathrm{Al} \star 80 \% \mathrm{Al}$
FIG. 1. High-angle annular dark field STEM micrographs of GaAs/ $\mathrm{Al}_{x} \mathrm{Ga}_{1-x} \mathrm{As}$ core/shell nanowires in cross-section. From left to right, $x=25 \%, 50 \%$, and $70 \%$. The darkest regions near the edges correspond to $\mathrm{Al}$-rich areas. In each of the images, we indicate the Al content in some representative regions as determined using energy-dispersive X-ray spectroscopy (EDX). detector. The nanowires were lying on a silicon substrate and probed in back-scattering geometry as depicted schematically in the inset of Figure 2. In order to obtain a high signal intensity, the incident light was polarized along the nanowire axis. The scattered light was not analyzed for its polarization. According to Raman selection rules, in the back-scattering configuration on $\{110\}$ surfaces, the LO phonon is forbidden, while the TO phonon is allowed. Finite-difference timedomain (FDTD) simulations of the electromagnetic field distribution in the nanowires were performed with the simulation package Meep.$^{54}$ We have considered the hexagonal geometry of the nanowires and the different dielectric constants of the core and shell, taken from Ref. 55.

\section{RESULTS AND DISCUSSION}

Raman spectra of $\mathrm{GaAs} / \mathrm{Al}_{x} \mathrm{Ga}_{1-x} \mathrm{As}$ core/shell nanowires with $x=25 \%, 50 \%$, and $70 \%$ are reported in Figure 2 . Peaks corresponding to the optical phonons of the GaAs core and $\mathrm{Al}_{x} \mathrm{Ga}_{1-x} \mathrm{As}$ shell are identified. The TO mode from the GaAs core is at $265 \mathrm{~cm}^{-1}$, in good agreement with the bulk value. ${ }^{57}$ Due to the large mass difference between $\mathrm{Al}$ and $\mathrm{Ga}$, two sets of alloy modes are given for the $\mathrm{Al}_{x} \mathrm{Ga}_{1-x} \mathrm{As}$ shell, whose frequency depends on the Al content. The GaAs-like modes lie very close to those from GaAs, while the AlAs-like are within the range of the 350 and $400 \mathrm{~cm}^{-1}$. The alloy-related peaks are broader than those from GaAs, reflecting a higher degree of disorder. At a first glance, one can see that spectra of all samples are dependent on the excitation energy. Among the differences, we find changes in the relative intensities between the peaks and the frequency position of the AlAs and GaAs-like modes.

We start reporting on the relative changes in the intensity of the TO and LO modes. As mentioned above, following the Raman selection rules, the LO-related peaks should not be observed. In agreement with this, the LO mode from GaAs barely appears in all spectra, while this is not the case for the alloy-related LO modes. The shorter-range nature of alloy modes can contribute into a partial breaking in the selection rules. One should also consider that when the excitation source is close to the energy band-gap of the material, the exciton becomes the real intermediate state in the Raman process. This condition leads to resonant scattering and the intensity is enhanced. The enhancement is more important for the LO phonons as a consequence of the preferential interaction between the exciton and the longitudinal electric field induced by LO phonons. In general, we can see a higher ratio of the LO/TO intensities for conditions close to such a resonance. We can see this effect when measuring nanowires with $\mathrm{Al}_{x} \mathrm{Ga}_{1-x} \mathrm{As}$ shells containing $x \approx 25 \%$ and $x \approx 70 \%$ with, respectively, $647.1 \mathrm{~nm}$ and $520.8 \mathrm{~nm}$ wavelengths, corresponding to 1.92 and $2.38 \mathrm{eV}$.

We now turn to the differences of intensity between the GaAs(core) and $\mathrm{Al}_{x} \mathrm{Ga}_{1-x} \mathrm{As}$ (shell) related peaks, as well as to the frequency position of the latter when probing with different excitation wavelengths. The Raman peaks were fitted with convoluted Lorentzian functions. The corresponding frequencies extracted from the fits are reported in Table I. For the AlAs and GaAs-like peaks, we also report on the 


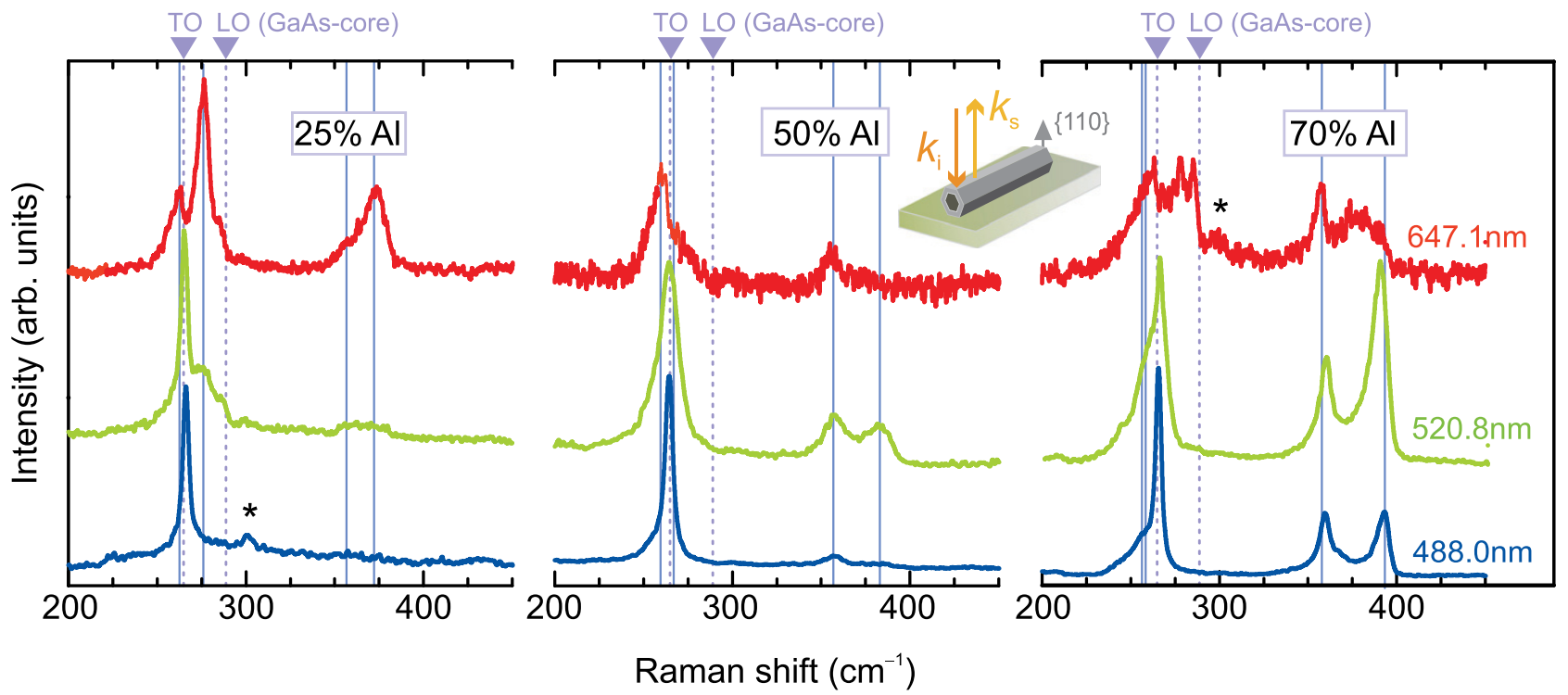

FIG. 2. Raman spectra of GaAs/ $\mathrm{Al}_{x} \mathrm{Ga}_{1-x} \mathrm{As}$ core/shell nanowires with $x=25 \%, 50 \%$, and $70 \%$ by using three different excitation wavelengths. The dotted (solid) lines correspond to the frequency position expected for the core (shell) modes. Silicon second order peak is indicated by a star. ${ }^{56}$

corresponding aluminum composition, as obtained from the expression given by Adachi. ${ }^{58}$ Due to the flat dispersion for the AlAs-like TO mode, we do not deduce any Al concentration from it.

Let us describe the spectra obtained for the nanowire with a $25 \% \mathrm{Al}$ in the shell. When we excite with $488.0 \mathrm{~nm}$, only the TO mode of GaAs is present. The spectrum does not show the existence of an $\mathrm{Al}_{x} \mathrm{Ga}_{1-x} \mathrm{As}$ shell. For longer wavelengths, other peaks appear, revealing the existence of the shell. This is a non-intuitive result, as in thin films one would expect to excite the parts closer to the surface with the shorter wavelengths. Exciting with $520.8 \mathrm{~nm}$, two peaks are observed: the most intense one corresponds to the TO mode of GaAs (core), while the less intense peak corresponds to a TO GaAs-like mode from the shell. There is an interesting change of the spectrum for the excitation with $647.1 \mathrm{~nm}$. Here, the AlAs and GaAs-like modes appear with a remarkably higher intensity. This increased scattering intensity is

TABLE I. Optical phonon energies as obtained from fits to the spectra in Fig. 2 and the relative alloy composition obtained from the expression given by Adachi. The fitting error was smaller than the experimental one $\left(0.7 \mathrm{~cm}^{-1}\right)$, except for the frequencies marked with a star. In such cases, the signal was weak and the error in the $\mathrm{Al}$ content is calculated from the fitting error. Otherwise it was obtained by using the experimental value. LVM stands for local vibrational mode.

\begin{tabular}{|c|c|c|c|c|c|c|c|c|c|}
\hline \multirow{2}{*}{$\begin{array}{l}\lambda \\
(\mathrm{nm})\end{array}$} & \multicolumn{3}{|c|}{$25 \% \mathrm{Al}$} & \multicolumn{3}{|c|}{$50 \% \mathrm{Al}$} & \multicolumn{3}{|c|}{$70 \% \mathrm{Al}$} \\
\hline & $\begin{array}{c}\text { Peak } \\
\left(\mathrm{cm}^{-1}\right)\end{array}$ & Mode & $\mathrm{Al} \%$ & $\begin{array}{c}\text { Peak } \\
\left(\mathrm{cm}^{-1}\right)\end{array}$ & Mode & $\mathrm{A} 1 \%$ & $\begin{array}{c}\text { Peak } \\
\left(\mathrm{cm}^{-1}\right)\end{array}$ & Mode & $\mathrm{A} 1 \%$ \\
\hline \multirow[t]{7}{*}{647.1} & 262.2 & TO,GaAs-like & $38 \pm 6$ & $255.1 *$ & TO,GaAs-like & $81 \pm 3$ & $248.5^{*}$ & GaAs-like & LVM \\
\hline & 276.0 & LO,GaAs-like & $27 \pm 2$ & 260.7 & TO,GaAs-like & $49 \pm 5$ & 261.3 & TO,GaAs-like & $45 \pm 5$ \\
\hline & 285.6 & LO,GaAs-like & $7 \pm 1$ & 273.4 & LO,GaAs-like & $33 \pm 2$ & 278.0 & LO,GaAs-like & $23 \pm 1$ \\
\hline & 361.7 & LO,AlAs-like & $7 \pm 1$ & 355.3 & TO,AlAs-like & $\ldots$ & 285.6 & LO,GaAs-like & $7 \pm 1$ \\
\hline & 373.5 & LO,AlAs-like & $26 \pm 1$ & $372.0 *$ & LO,AlAs-like & $23 \pm 3$ & 356.9 & TO,AlAs-like & $\ldots$ \\
\hline & & & & & & & 377.4 & LO,AlAs-like & $33 \pm 1$ \\
\hline & & & & & & & 389.9 & LO,AlAs-like & $60 \pm 2$ \\
\hline \multirow[t]{6}{*}{520.8} & $258.0 *$ & TO,GaAs-like & $66 \pm 4$ & 256.7 & TO,GaAs-like & $73 \pm 4$ & $243.2^{*}$ & GaAs-like & LVM \\
\hline & 265.3 & $T O, G a A s$ & core & 265.9 & $T O, G a A s$ & core & 257.3 & TO,GaAs-like & $70 \pm 4$ \\
\hline & 276.3 & LO,GaAs-like & $27 \pm 2$ & 358.9 & TO,AlAs-like & $\cdots$ & 265.3 & $T O, G a A s$ & core \\
\hline & 286.7 & LO,GaAs-like & $5 \pm 1$ & 383.8 & LO,AlAs-like & $46 \pm 1$ & 358.6 & TO,AlAs-like & $\ldots$ \\
\hline & $358.0^{*}$ & TO,AlAs-like & $\ldots$ & & & & 383.3 & LO,AlAs-like & $45 \pm 1$ \\
\hline & $373.4^{*}$ & LO,AlAs-like & $26 \pm 4$ & & & & 389.8 & LO,AlAs-like & $60 \pm 2$ \\
\hline \multirow[t]{7}{*}{488.0} & 266.1 & $T O, G a A s$ & core & 265.0 & $T O, G a A s$ & core & $247.0 *$ & GaAs-like & LVM \\
\hline & & & & $358.2 *$ & TO,AlAs-like & $\cdots$ & 257.3 & TO,GaAs-like & $70 \pm 4$ \\
\hline & & & & & & & 265.7 & TO,GaAs & core \\
\hline & & & & & & & 359.6 & TO,AlAs-like & $\ldots$ \\
\hline & & & & & & & $363.7^{*}$ & LO,AlAs-like & $10 \pm 1$ \\
\hline & & & & & & & $389.9 *$ & LO,AlAs-like & $60 \pm 2$ \\
\hline & & & & & & & 393.6 & LO,AlAs-like & $71 \pm 2$ \\
\hline
\end{tabular}


explained by approaching resonance conditions. In fact, this excitation wavelength corresponds to the resonance condition of $\mathrm{Al}_{x} \mathrm{Ga}_{1-x} \mathrm{As}$ with $x \approx 35 \%$. The positions of the LO GaAs and AlAs-like peaks are consistent with an Al composition of 26-27\%, while the TO GaAs-like peaks suggest a higher $\mathrm{Al}$ composition (38\%). As a general trend, the $\mathrm{Al}$ content obtained from TO is higher than LO. We attribute this to a slight difference in the dependence of the TO and the LO GaAs-like modes as a function of the stoichiometry of the $\mathrm{Al}_{x} \mathrm{Ga}_{1-x}$ As.

We turn now to the nanowires with $50 \% \mathrm{Al}$ in the $\mathrm{Al}_{x} \mathrm{Ga}_{1-x} \mathrm{As}$ shell. Also here, the signal from the GaAs core is mostly prominent when probed with the short wavelength $(488.0 \mathrm{~nm})$. The spectrum obtained with $520.8 \mathrm{~nm}$ excitation contains information on the core and the shell. In contrast, when we excite with $647.1 \mathrm{~nm}$, mostly only the TO peaks related to the shell are present. Here, the TO GaAs-like peaks suggest an $\mathrm{Al}$ concentration around $50 \%$. Interestingly, we observe a peak consistent with $33 \% \mathrm{Al}$ when exciting with $647.1 \mathrm{~nm}$. We attribute this to the existence of inhomogeneities in the shell as reported in Figure 1 and in agreement with what has been found in the past in similar structures. Indeed, previous studies ${ }^{26}$ have shown that there is segregation of $\mathrm{Al}$ in $\{112\}$ apex of hexagonal section. Moreover, in the $\{110\}$ facets, there is an additional variation in composition at the nanometer scale. Even though these regions are in the order of few nanometers, the resonance conditions enable us to detect them in an enhanced manner.

To complete the picture, we comment on the measurements performed on the nanowire with an $\mathrm{Al}_{x} \mathrm{Ga}_{1-x} \mathrm{As}$ shell of $x=70 \%$. In addition to the variation of the signal intensity between core and shell as a function of excitation wavelength, we also observe a marked change in the frequency position of the shell related peaks. Since it is difficult to distinguish the GaAs-like TO and LO modes, we consider not appropriate to extract the concentration from one of the two curves, while it is possible to fit one peak, revealing their character of localized mode of an isolated Ga atom in AlAs. The position of the LO AlAs-like peak is consistent with a concentration of aluminum of $x=70 \%$, in the spectrum obtained with $488.0 \mathrm{~nm}$ wavelength, while we find the LO AlAs-like mode corresponding to $60 \% \mathrm{Al}$ content when probed with $520.8 \mathrm{~nm}$. In fact, this is consistent with the $520.8 \mathrm{~nm}$ wavelength being in resonance with an $\mathrm{Al}_{x} \mathrm{Ga}_{1-x} \mathrm{As}$ of $x \approx 65 \%$. The spectra obtained with the $647.1 \mathrm{~nm}$ wavelength are richer in peaks. The different observed peaks are consistent with different $\mathrm{Al}$ concentrations between $7 \%$ and $60 \%$. Similar concentrations are obtained for both the GaAs and AlAs-like modes. As for the previous nanowires, the different $\mathrm{Al}$ concentrations are attributed to the fluctuation of the $\mathrm{Al}$ composition in the shell.

Finally, we address the differences in the spectra as a function of the excitation wavelength by considering the distribution of the photonic modes within the nanowire as a function of the excitation wavelength. It is well-known that the Raman scattering intensity is directly linked to the local internal electric field of the excitation. ${ }^{43}$ In order to assess the distribution of the radiation in the nanowires, we have performed FDTD simulations considering the exact hexagonal geometry of the nanowire and the dielectric constants of the core and shell. We have simulated a plane wave polarized along the nanowire axis. We have reported in Supporting Information an animation of the electric field energy density as a function of the time under periodic steady state. ${ }^{51}$ Figure 3 shows the cross-sectional maps of the time-averaged electric field energy density obtained for a nanowire lying on a $\mathrm{Si} / \mathrm{SiO}_{2}$ substrate during illumination from a top monochromatic light source. Clearly, light distributes unevenly across the nanowire cross-section. The simplest case corresponds to the nanowires with a $25 \%$ of $\mathrm{Al}$ in the shell. The simulations show a gradual increase in the field in the shell by increasing wavelength. However, the field in the core is always the most intense. This is because of the small size of the nanowire. The simulations match well with the experiments, where an increase in the peaks related to the shell is observed by increasing wavelength. We must note here that for the $647.1 \mathrm{~nm}$ excitation probing the Raman, intensities of the peaks related to the

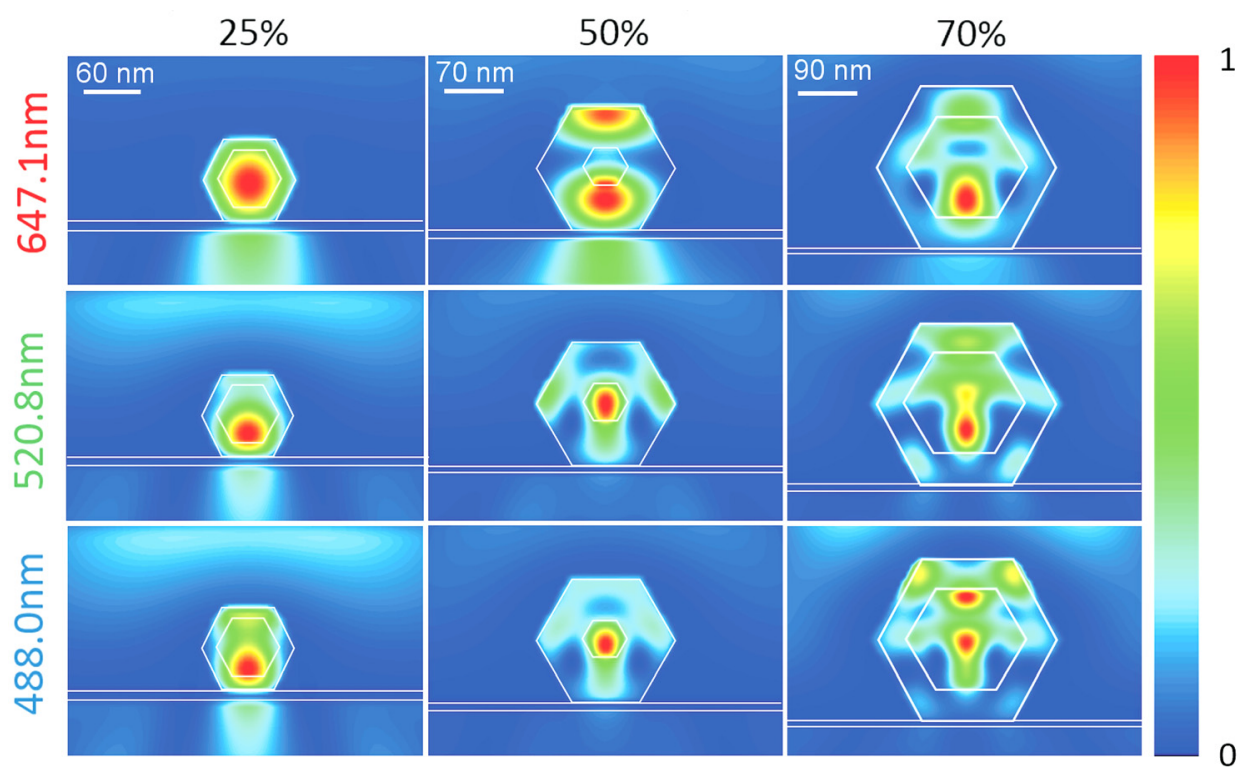

FIG. 3. Simulated cross-sectional maps of the internal field energy density distribution given by a top plane wave incoming onto GaAs/ $\mathrm{Al}_{x} \mathrm{Ga}_{1-x} \mathrm{As}$ core/shell nanowire system, as described in the manuscript. From bottom to top, the fields correspond to a excitation wavelength of 488.0, 520.8, and $647.1 \mathrm{~nm}$, respectively. 
shell are also intensified because of the near resonance conditions. In the case of the nanowires with $50 \% \mathrm{Al}$ in the shell, the light field is more intense in the core $(\mathrm{GaAs})$ for an incoming wavelength of $488.0 \mathrm{~nm}$, while light becomes distributed around the shell when using longer wavelengths. These results are in excellent agreement with our experiments, where the core-related Raman signal is almost not present when exciting with the $647.1 \mathrm{~nm}$ wavelength. Finally, in the nanowire with $x=70 \%$, there is no obvious trend. It must be noted that at short wavelength, the local field energy around the shell is confined at the edges of the nanowire cross-section where the $\mathrm{Al}$ content is found the largest. This would be in agreement with the observation of an increasing $\mathrm{Al}$ content from Raman spectra with excitation energy. However for this sample, the photonic effects are in harmony with an increase in the Raman intensity of area with high $\mathrm{Al}$ content due to selective resonance. Consequently, we cannot discern the two effects here.

\section{CONCLUSION}

As a conclusion, we have shown that a complete chemical characterization of core-shell nanowire structures requires the probing with different wavelengths as well as simulations of the photonic modes existing in the nanowires for the different excitation wavelengths. The multi-spectral characterization enables the probing of different parts of the cross-section as well as the local inhomogeneities in chemical composition.

\section{ACKNOWLEDGMENTS}

F.A. and A.F.M. thank funding from SNF through Project No. 137648. E.A.L. acknowledges funding from Marie Curie Actions Program. A.F.M. thanks funding through the ERC Starting Grant UpCon and NCCR-QSIT. J.A. acknowledges the funding from the Generalitat de Catalunya 2014 SGR 1638. M.d.1.M. thanks the CSIC JaePredoc program.

${ }^{1}$ X. Duan, Y. Huang, R. Agarwal, and C. Lieber, Nature 421, 241 (2003). ${ }^{2}$ P. Krogstrup, H. Jorgensen, M. Heiss, O. Demichel, J. Holm, M. Aagesen, J. Nygard, and A. Fontcuberta i Morral, Nat. Photonics 7, 306 (2013).

${ }^{3}$ C. Soci, A. Zhang, B. Xiang, S. A. Dayeh, D. Aplin, J. Park, X. Bao, Y. H. Lo, and D. Wang, Nano Lett. 7, 1003 (2007).

${ }^{4}$ E. C. Garnett, M. L. Brongersma, Y. Cui, and M. D. McGehee, Annu. Rev. Mater. Sci. 41, 269 (2011).

${ }^{5}$ E. Garnett and P. Yang, Nano Lett. 10, 1082 (2010).

${ }^{6}$ M. D. Kelzenberg, S. W. Boettcher, J. A. Petykiewicz, D. B. TurnerEvans, M. C. Putnam, E. L. Warren, J. M. Spurgeon, R. M. Briggs, N. S. Lewis, and H. A. Atwater, Nature Mater. 9, 368 (2010).

${ }^{7}$ J. R. Maiolo, B. M. Kayes, M. A. Filler, M. C. Putnam, M. D. Kelzenberg, H. A. Atwater, and N. S. Lewis, J. Am. Chem. Soc. 129, 12346 (2007).

${ }^{8}$ Y. Cui, Q. Wei, H. Park, and C. M. Lieber, Science 293, 1289 (2001).

${ }^{9}$ M. S. Gudiksen, L. J. Lauhon, J. Wang, D. C. Smith, and C. M. Lieber, Nature Mater. 415, 617 (2002)

${ }^{10}$ D. Saxena, S. Mokkapati, P. Parkinson, N. Jiang, Q. Gao, H. H. Tan, and C. Jagadish, Nat. Photonics 7, 963 (2013).

${ }^{11}$ Y. Wang, V. Schmidt, S. Senz, and U. Gösele, Nat. Nanotechnol. 1, 186 (2006).

${ }^{12}$ Y. Xiang, L. Cao, S. Conesa-Boj, S. Estrade, J. Arbiol, F. Peiro, M. Heiss, I. Zardo, J. R. Morante, M. L. Brongersma et al., Nanotechnology 20, 245608 (2009).
${ }^{13}$ B. Mandl, J. Stangl, T. Martensson, A. Mikkelsen, J. Eriksson, L. S. Karlsson, G. Bauer, L. Samuelson, and W. Seifert, Nano Lett. 6, 1817 (2006).

${ }^{14}$ C. J. Novotny and P. K. L. Yu, Appl. Phys. Lett. 87, 203111 (2005).

${ }^{15}$ E. A. Stach, P. J. Pauzauskie, T. Kuykendall, J. Goldberger, R. He, and P. Yang, Nano Lett. 3, 867 (2003).

${ }^{16}$ C. Colombo, D. Spirkoska, M. Frimmer, G. Abstreiter, and A. Fontcuberta i Morral, Phys. Rev. B 77, 155326 (2008).

${ }^{17}$ C. P. T. Svensson, W. Seifert, M. W. Larsson, L. R. Wallenberg, J. Stangl, G. Bauer, and L. Samuelson, Nanotechnology 16, 936 (2005).

${ }^{18}$ K. A. Dick, S. Kodambaka, M. C. Reuter, K. Deppert, L. Samuelson, W. Seifert, L. R. Wallenberg, and F. M. Ross, Nano Lett. 7, 1817 (2007).

${ }^{19}$ N. Zakharov, P. Werner, G. Gerth, L. Schubert, L. Sokolov, and U. Gösele, J. Cryst. Growth 290, 6 (2006).

${ }^{20}$ J. Noborisaka, J. Motohisa, S. Hara, and T. Fukui, Appl. Phys. Lett. 87, 093109 (2005).

${ }^{21}$ F. Qian, M. Brewster, S. K. Lim, Y. Ling, C. Greene, O. Laboutin, J. W. Johnson, S. Gradečak, Y. Cao, and Y. Li, Nano Lett. 12, 3344 (2012).

${ }^{22}$ F. Qian, S. Gradečak, Y. Li, C. Wen, and C. Lieber, Nano Lett. 5, 2287 (2005).

${ }^{23}$ F. Qian, Y. Li, S. Gradečak, D. Wang, C. J. Barrelet, and C. M. Lieber, Nano Lett. 4, 1975 (2004).

${ }^{24}$ M. Fickenscher, T. Shi, H. E. Jackson, L. M. Smith, J. M. Yarrison-Rice, C. Zheng, P. Miller, J. Etheridge, B. M. Wong, Q. Gao et al., Nano Lett. 13, 1016 (2013).

${ }^{25}$ C. Zheng, J. Wong-Leung, Q. Gao, H. H. Tan, C. Jagadish, and J. Etheridgel, Nano Lett. 13, 3742 (2013).

${ }^{26}$ M. Heiss, Y. Fontana, A. Gustafsson, G. Wüst, C. Magen, D. D. O. Regan, J. W. Luo, B. Ketterer, S. Conesa-Boj, A. V. Kuhlmann et al., Nature Mater. 12, 439 (2013).

${ }^{27}$ D. E. Perea, E. R. Hemesath, E. J. Schwalbach, J. L. Lensch-Falk, P. W. Voorhees, and L. J. Lauhon, Nat. Nanotechnol. 4, 315 (2009).

${ }^{28}$ P. Parayanthal, F. H. Pollak, and J. M. Woodall, Appl. Phys. Lett. 41, 961 (1982).

${ }^{29}$ S. Hernandez, R. Cusco, D. Pastor, L. Artus, K. P. O. Donnell, R. W. Martin, I. M. Watson, Y. Nanishi, and E. Calleja, J. Appl. Phys. 98, 013511 (2005).

${ }^{30}$ E. Alarcon-Llado, S. Conesa-Boj, X. Wallart, P. Caroff, and A. Fontcuberta i Morral, Nanotechnology 24, 405707 (2013).

${ }^{31}$ Y. S. Chen, W. Shockley, and G. L. Pearson, Phys. Rev. 151, 648 (1966).

${ }^{32}$ P. M. Mooney, F. H. Dacol, J. C. Tsang, and J. O. Chu, Appl. Phys. Lett. 62, 2069 (1993).

${ }^{33}$ I. Zardo, S. Conesa-Boj, F. Peiro, J. R. Morante, J. Arbiol, E. Uccelli, G. Abstreiter, and A. Fontcuberta i Morral, Phys. Rev. B 80, 245324 (2009).

${ }^{34}$ G. Signorello, E. Lortscher, P. Khomyakov, S. Karg, D. Dheeraj, B. Gotsmann, H. Weman, and H. Riel, Nat. Commun. 5, 3655 (2014).

${ }^{35}$ G. Signorello, S. Karg, M. Björk, B. Gotsmann, and H. Riel, Nano Lett. 13, 917 (2013).

${ }^{36}$ T. Livneh, J. Zhang, G. Cheng, and M. Moskovits, Phys. Rev. B 74, 035320 (2006).

${ }^{37}$ G. Chen, J. Wu, Q. Lu, H. R. Gutierrez, Q. Xiong, M. E. Pellen, J. S. Petko, D. H. Werne, and P. C. Eklund, Nano Lett. 8, 1341 (2008).

${ }^{38}$ J. Wang, M. S. Gudiksen, X. Duan, Y. Cui, and C. M. Lieber, Science 293, 1455 (2001).

${ }^{39}$ X. Wang, C. J. Summers, and Z. L. Wang, Nano Lett. 4, 423 (2004).

${ }^{40}$ L. Cao, P. Fan, A. P. Vasudev, J. S. White, Z. Yu, W. Cai, J. A. Schuller, S. Fan, and M. L. Brongersma, Nano Lett. 10, 439 (2010).

${ }^{41}$ L. Cao, J. White, J. Park, J. Schuller, B. M. Clemens, and M. Brongersma, Nature Mater. 8, 643 (2009).

${ }^{42}$ S. A. Mann and E. Garnett, Nano Lett. 13, 3173 (2013).

${ }^{43}$ L. Cao, B. Nabet, and J. Spanier, Phys. Rev. Lett. 96, 157402 (2006).

${ }^{44}$ F. J. Lopez, J. K. Hyun, U. Givan, I. S. Kim, and A. Holsteen, Nano Lett. 12, 2266 (2012)

${ }^{45}$ E. Kim, A. Steinbruck, M. T. Buscaglia, V. Buscaglia, T. Pertsch, and R. Grange, ACS Nano 7, 5343 (2013).

${ }^{46}$ F. Dutto, C. Raillon, K. Schenk, and A. Radenovic, Nano Lett. 11, 2517 (2011).

${ }^{47}$ F. Dutto, M. Heiss, A. Lovera, O. Lopez-Sanchex, A. Fontcuberta i Morral, and A. Radenovic, Nano Lett. 13, 6048 (2013).

${ }^{48}$ E. Uccelli, J. Arbiol, C. Magen, P. Krogstrup, E. Russo-Averchi, M. Heiss, G. Mugny, F. Morier-Genoud, J. Nygard, J. Morante et al., Nano Lett. 11, 3827 (2011)

${ }^{49}$ E. Russo-Averchi, M. Heiss, L. Michelet, P. Krogstrup, J. Nygard, C. Magen, J. R. Morante, E. Uccelli, J. Arbiol, and A. Fontcuberta i Morral, Nanoscale 4, 1486 (2012). 
${ }^{50}$ M. Heigoldt, J. Arbiol, D. Spirkoska, J. M. Rebled, S. Conesa-Boj, G. Abstreiter, F. Peiró, J. R. Morante, and A. Fontcuberta i Morral, J. Mater. Chem. 19, 840 (2009).

${ }^{51}$ See supplementary material at http://dx.doi.org/10.1063/1.4901504 for the growth protocol of the nanowires; the animation of the internal field energy density as a function of time under steady state.

${ }^{52}$ D. C. Watson, R. V. Martinez, Y. Fontana, E. Russo-Averchi, M. Heiss, A. Fontcuberta i Morral, G. M. Whitesides, and M. Loncar, Nano Lett. 14, 524 (2014).
${ }^{53}$ L. Ouattara, A. Mikkelsen, N. Sköld, J. Eriksson, T. Knaapen, E. Cavar, W. Seifert, L. Samuelson, and E. Lundgren, Nano Lett. 7, 2859 (2007).

${ }^{54}$ A. F. Oskooi, D. Roundy, M. Ibanescu, P. Bermel, J. Joannopoulos, and S. G. Johnson, Comput. Phys. Commun. 181, 687 (2010).

${ }^{55}$ D. E. Aspes, S. M. Kelso, R. Logan, and R. Bhat, J. Appl. Phys. 60, 754 (1986).

${ }^{56}$ C. S. Wang, J. Chen, R. Becker, and A. Zdetsis, Phys. Lett. A 44, 517 (1973).

${ }^{57} \mathrm{P}$. Yu and M. Cardona, Fundamentals of Semiconductors (Springer, Berlin, 1996).

${ }^{58}$ S. Adachi, J. Appl. Phys. 58, R1 (1985). 